His hereditary tendencies become important. Heredity, in spite of many efforts to diminish if not to extinguish its importance, is still generally admitted to influence disease in man, and $I$ incline to think that it operates rather in a negative than a positive way. That is, assuming power of resistance, inheritances tend to produce points of nonresistance; or, to put it in another way, inheritance is rather the weakening in a particular direction of an inherent good quality than the intensifying of an evil one. Perhaps it may be said it is the same thing; to me, however, it is a more comfortable and hopeful doctrine.

The mention of the individual brings me back to the question, How is the resistance natural to every man to be evoked?

I take it, if we accept the doctrine of natural resistance, this is really the object of all our treatment of disease; we endeavour by our management of the case to bring out all the forces, whatever they be, that lie dormant in every one, and assist their operation either by directly strengthening them or by lessening any obstacles to their beneficent action.

But just here it is that we are still on uncertain ground, and until we know these things our treatment must be more or less empirical. Light is coming but slowly. The modern treatment of disease by antitoxins and vaccines is surely based upon the principle of helping Nature by the indirect action of living forces. Yet even here our path is beset with dangers, as evidenced by the difficulties of what is known as anaphylaxis.

And even if we knew Nature's general scheme of resistance to disease, even if we solved all the problems that I have mentioned as well as the numerous others that I have omitted, there would still remain the difficulty of the individual; for, as I said before, every man is an individual, and even at the best more or less imperfect. Perfection, whether of mind or body, is hardly to be looked for. We have, therefore, to take our patients as we find them, with all their imperfections, their idiosyncrasies of temperament, their weaknesses, whether due to themselves or their inheritances, and do our best to help them to recover from disease by strengthening the powers for good that remain to them. Alas, how often no response is made to our efforts! From one cause or another the power of resistance has gone, and we can only look on as the case progresses to a fatal termination. The maxim, "Treat the patient and not his disease," has a solid truth behind it, and if we knew our patients better in their ordinary lives and in their earlier slight departures from health, we should be in a better position to treat them in disease. Unfortunately we do not very often get the opportunity, but when we do, what an incalculable advantage it is! and if people only realized this, much could be done. If the prevention of disease is one of our highest aims, an important part of it must consist in the preservation of the health of the individual by teaching him how to keep his defences in good order.

Lastly, I think we must all realize that medicine is still far from being an exact science and that our ignorance is greater than our knowledge. But, if we also realized that the body is endowed with large powers of resistance to clisease and that the problem of treatment is how to evoke those powers and help their utilization to the best advantage, and if we realized that resistance varies in the individual, then we would recognize that there is a certain unity in medicine, that it is the treatment of disease and not of diseases; we would recognize that we are not the masters but the servants of Nature, and we would sit humbly at her feet patiently investigating the secrets of that most marvellous collection of forces, the living human body, trying to help her, not endeavouring to take the case out of her hands. And a recognition of the unity of medicine would promote a spirit of unity among ourselves, we should hear less of systems of treatment, we should be spared those unedifying controversies in regard to special methods, of which we have had several instances lately, and which I confess are to me painful, for we would recognize that the goal we are all seeking to attain can be reached by several routes, and that no one of us has a monopoly of wisdom.

And, though medicine is somewhat overshadowed by the triumphs of her more brilliant sister, surgery, may we not look forward to a time when, by a sound application of the knowledge revealed to us by a patient investigation of
Nature's methods of dealing with disease, we shall regain for medicine a portion of the field which of late years she has been forced to relinquish?

REFERENCE.
Sir Watson Cheyne, Wightman Lecture, 1908.

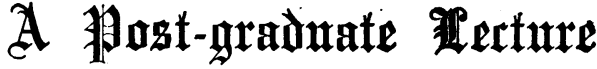

\section{THE DIAGNOSIS OF SPINAL CORD AFFECTIONS.}

By DAVID FORSYTH, M.D., D.Sc., F.R.C.P., CHARING CROSS AND EVELINA HOSPITALS.

IF an account of the ordinary types of some everyday diseases is not too jejune for a post-graduate audience, I will, without parading anything rare or anomalous or even exceptional, review the common affections of the spinal cord. From the clinical standpoint, these cases arrange themselves in three groups according to their onset: (1) Those in which the symptoms come on all at once; (2) those in which the symptoms are acute, taking several hours or even days to develop; (3) those in which the symptoms are insidious.

I. When the Symptoms Begin All At Once.

So few affections begin in this way that the diagnosis is rarely involved, and, as a matter of fact, we have practically only two lesions to think of-trauma and haemorrhage.

In trauma, leading to injury of the cord, often as a result of fracture-dislocation, the diagnosis is presented to us in the history either of direct violence to the spine (as in falling from a height or from stabs and bullet wounds) or of indirect violence (by a weight hitting the head or shoulders or by striking the head passing under an arch). In these cases, if the patient-lives, a question that may arise is the distinction between organic injury and mere concussion of the spine; with the latter, however, the patient should rapidly improve until, after a few days, his recovery is assured."

Haemorrhage (haematomyelia), apart from injury, is likely to occur only if the cord is already softened by myelitis or a new growth; and though, in either event, the bleeding produces its effects within a few minutes, some history of the older-standing trouble is sure to be forthcoming. Yet another possible cause of haemorrhage that must not be forgotten is one of the blood diseases, especially pernicious anaemia. Whatever the cause, however, the symptoms by which haematomyelia is to be recognized depend on the position and size of the effusion. As a rule, we find that, without warning, the patient quickly becomes paralysed and anaesthetic up to the level of the lesion. Further, by the inevitable destruction, locally, of the motor nerve cells, the corresponding muscles atrophy and show the reaction of degeneration. Of course, in some cases, the haemorrhage, instead of lying inside the cord, is meningeal (as, for example, when an aneurysm bursts into the spinal canal), and on these occasions the symptoms, as sudden as before, indicate at first irritation of the nerve roots (paroxysmal shooting pains along the sensory fibres, with twitching or spasm of the muscles), and, later, compression of the cord by the effused blood. At this stage, when all impulses, up and down, are interfered with, the patient's sensation of every kind below the lesion is numbed or lost, while his muscles are weakened or paralysed.

\section{When the Symptoms Begin Acutely.}

In these cases we find, as a rule, that the patients, so far at any rate as their nervous system is concerned, felt quite well until a day or two before, but that since then their nervous symptoms have come on rapidly. In these circumstances the diagnosis lies between three conditions : (1) An acute infective process in the cord; (2) some thrombosis in the spinal vessels; (3) acute spinal

* "Railway spine" - really a neurasthenic condition, better called "railway brain," as Thorburn suggested-- presents rather 
meningitis. Truly, two other conditions-Landry's paralysis, which spreads within a few days from legs to trunk, to arms, to neck and head, when the patient dies, and diver's paralysis (caisson disease), overtaking workers in high-pressure chambers-might claim admission in this group, but they are in the one instance so rare and in the other so readily diagnosed by the suggestive history as to require no further time spent on them.

\section{An Acute Infective Process.}

These infections, inflammatory or toxic, attack either the grey and white matter together or the grey matter alone; in the former case we have acute myelitis, in the latter acute poliomyelitis. Both of them, being infective, are likely to be ushered in by febrile symptoms-headache, malaise, a little temperature, and so on. The nervous symptoms themselves depend, of course, as to their details, on the position of the inflammation.

Myelitis most often picks out the dorsal cord-sometimes the cervical, sometimes the lumbar. Sometimes, even, it is scattered up and down the cord (and in the brain as well) like, as it were, an acute form of disseminated sclerosis. Whatever its level, however, it usually spreads across the cord as a transverse lesion. This means that with every tract, ascending and descending, being involved at that level, all the nervous conditions lower down-sensory, motor, and reflex-will be disturbed. The patient, therefore, rapidly loses the power in his legs, which, for the time being, become flaccid; his sensation of every kind is dulled or lost; his reflexes, after disappearing for a while, return, become exaggerated, and, with ankle-clonus and Babinski's sign, mark the gradual onset of muscular rigidity.* Often, too, the upper limit of the anaesthesia is fringed by hyperaesthesia. In harmony with these changes the bladder as well as the rectum passes out of control, and the urine is retained to overflowing.

These are the results of the commonest form of myelitis -a dorsal lesion. What are the differences if the cervical cord is attacked? In addition to the foregoing, there is paralysis of the arms and intercostals, with anaesthesia running up to the new level. In the highest cervical lesions of all, the neck muscles, as well as the arm, may be paralysed; and the same with the diaphragm. Moreover, whatever the position of the rnyelitis, the nerve cells at that level will be destroyed; so that, in a case with the cervical or lumbar enlargement affected, we shall get not only paralysis, but muscular atrophy in the arms or legs, as the case may be, with the reaction of degeneration and the permanent loss of the tendon reflexes in the limb.

With myelitis in its disseminated form the effects, for the most part, are the same, but are distributed irregu larly and, perhaps, perplexingly, more especially as some cerebral symptoms, such as nystagmus or optic neuritis, may be produced.

Acute Poliomyelitis.-The other acute infective process needs very few words to fill in its diagnostic features. Common only in children, it directly attacks nothing of importance except the motor cells, and therefore its direct effects are seen nowhere except in muscular paralysis. In its clinical course, as its ephemeral fever subsides (a fever so mild as sometimes to be overlooked altogether), the child is found to be paralysed-one limb, two limbs, all four limbs. The corresponding reflexes are gone andthat is all; unless, indeed, it be that the child complains of some pain when its limbs are handled. Later, of course-perhaps at the end of the week-electrical changes will appear with probably enough muscular atrophy to be both seen and felt; but, on the other hand, by this time some at any rate of the original damage should be made good, and, instead of, say, four paralysed limbs, the child now dangles only one.

\section{Spinal Thrombosis.}

This the second condition responsible for acute symptoms, is the result, apart from pathological states of the blood itself, of arterial degeneration, which, narrowing the vessels, hinders the stream of blood until it clots. As soon as this happens, that part of the cord deprived of its nourishment rapidly degenerates-a sequence of events

These changes do not occur if the transverse lesion is completethat is, destroys every fibre and cell at the level. Instead, we have flaccidity with permanently lost reflexes. better known in connexion with the analogous condition of cerebral thrombosis leading up to cerebral softening. Moreorer, in the cord as in the brain, the outstanding cause of this endarteritis is syphilis, so that, for all practical purposes, we can interpret spinal thrombosis as syphilitic thrombosis. Occasionally, however, in old people, senile arterio-sclerosis acts in the same way.

As we might suppose from the nature of the process, its sum effect on the nervous elements of the cord is not very dissimilar to that of acute myelitis. Indeed, so alike are they that syphilitic thrombosis is often referred to, even at the present day, as syphilitic myelitis. Still, the former term is in every way preferable, even though, by their symptoms alone, thrombosis and myelitis can hardly be separated. To do this we are generally thrown back on any circumstantial evidence of syphilis (including the Wassermann reaction) that we can find; and the search for this needs to be closely pressed because of the necessity, before venturing a prognosis or beginning the treatment, of disentangling the two conditions.

Just one word more before leaving this question of syphilis and the cord. In addition to exciting thrombosis, the spirochaetes are guilty of other offences against the cord. Thus, their effects sometimes fall hearily on the spinal meninges, with their sensitiveness to pathological organisms, and, as a matter of fact, syphilitic meningitis is, perhaps, even commoner than thrombosis, though, most often, cord and meninges are affected together. This is the condition known as " meningo-myelitis." Yet here again the main trouble is vascular arteritis and phlebitisand the blood clots both in the spinal and meningeal vessels. Even before this, however, the pia arachnoid is inflamed by a gummatous infiltration, and is sometimes studded with little miliary gummata. Though these morbid changes take time, the symptoms they produce may begin acutely at any time from a few months to several years after the infection. Once set in train, the earliest of them are meningeal, resulting from spinal nerveroot irritation-that is to say, shooting and girdle pains, hyperaesthesia, and perhaps muscular spasm, followed later by paralysis and anaesthesia, but still pain. Finally, as the arterial disease makes itself felt in the cord itself, we get back to our clinical picture of spinal thrombosis.

Throughout the opening stages of this meningo-myelitis, the diagnosis of spinal root irritation may be easy enough; the difficulty is to identify the meningitis as syphilitic. In addition to the Wassermann reaction, some help can be found, as will be explained immediately, in the cytology of the cerebro-spinal fluid, though, even with this, we must again remember to look for corroborative evidence in other directions.

\section{Acrite Spinal Meningitis.}

This, the last of our groups of acute spinal affections, must, in order to fit the limits of this lecture, first be whittled down to get rid of those varieties of meningitis which, clinically, appear rather as cerebral cases than spinal. Thus we can put on one side tuberculous meningitis, septic, pneumococcal, influenzal, post-basal, gonococcal meningitis. All of these, no doubt, are acute infections of the spinal meninges, and yet none of them can fairly be made to stand as a disease of the spinal cord. Nor, for that matter, can spotted fever, although we call it cerebro-spinal meningitis. Still, before letting this one go, a small item of information can be extracted from it-namely, the connexion between spinal meningitis and head retraction, as seen so often in babies. Sometimes the explanation is offered that, with the head retracted, the cerebro-spinal fluid finds more room at the base of the brain, thereby relieving, to this extent, the increased cerebro-spinal pressure. The valid explanation, however, is of another sort. The head is retracted by muscles at the back of the neck (especially the complexus, the splenius capitis, and the upper part of the trapezius), which are innervated by the upper cervical spinal nerves. If, therefore, these roots are irritated, no matter what the cause, these muscles will be thrown into spasm, and will draw the head back. Head retraction, therefore, signifies cervical root irritation; and the most striking cause of this is cerebro-spinal meningitis.

To return to our muttons, however. The only truly spinal forms of meningitis are, first, acute septic meningitis resulting, as a rule, from inflammation spreading to the 
membranes from a neighbouring part, and, secondly, the syphilitic meningitis already described. Sometimes, with the first-named, the attempt is made to distinguish a pachymeningeal from a leptomeningeal form-a distinction, however, without any clinical value, even if it can be made post-mortem. Practically always, if the dura is inflamed, the pia arachnoid is inflamed as well; and, even if an exception occurred, we are quite without the clinical means of separating the two. Acute spinal meningitis, then, so far as we are concerned, is in one kind only. The cases are not very common, but usually the patient, perhaps after a rigor, soon develops the sensory and motor signs, already described, of root irritation, which, at first, may be localized to a few spinal segments. Later, however, if the infection spreads over the cord, the symptoms keep pace witli it until, finally, the patient passes into a condition, distressing to witness, which at first sight might even be mistaken for tetanus. He is thrown into painful opisthotonos by the spasms of his back muscles, while at the same time his legs and arms go stiff and rigid. Worse still, his suffering is aggravated; partly by his acutely hyperaesthetic skin, but more by the harrowing, darting pains that shoot along his spinal nerves. Towards the end, however, he finds a merciful relief in the paralysis which follows the irritation, and the spasms give place to flaccidity, the pain to anaesthesia. In these cases, of course, before settling the diagnosis, one would expect to find the primary source of the infection; and this most often lies in a carious vertebra, though sometimes in bedsores, local abscesses, cellulitis, or rarely, infective endocarditis.

So far as the organism responsible for a spinal meningitis is concerned, the point can hardly be settled without a bacteriological examination after a lumbar puncture. Even, however, in answering the main question-Is it meningitis? - the cerebro-spinal fluid is a great help, because when the meninges are inflamed great numbers of cells find their way into it together with inflammatory plasma. If, therefore, the fluid is turbid from cells, or if it gives chemical tests showing an excess of albumen, we have two pretty sure signs of meningitis, whatever its cause. Further, if the cells are polynuclear, the meningitis is very acute (such as the septic form we have been considering), but, if they are mainly lymphocytes, it is a milder variety (syphilitic, for example).*

III. When the Symptoms Begin Insidiously.

In this, our last group, the patients, as a rule, cannot say exactly how long they have been ill. All they know is that their symptoms, too trivial at first to be serionsly heeded, have gradually obtruded themselves until at last they- are beginning to interfere with their daily affairs. Now, as this insidious onset is shared by more than half a dozen chronic spinal affections, we must keep at hand some scheme of investigation by the help of which we can, in any given case and without waste of time, put aside the majority of the chronic affections, leaving only the two or three between which the diagnosis lies.

To do this, let us first mentally divide the whole group into two-those with none but motor symptoms, and those with sensory symptoms (whether with or without motor symptoms). Among the former we can place:

$$
\text { Motor Symptoms Only. }
$$

Progressive muscular atrophy-that is, chronic poliomyelitis.

Amyotrophic lateral sclerosis.

Primary spastic paraplegia.

Friedreich's ataxy.

Ataxic paraplegia.

And among the others:

Tabes.

$$
\text { Sensory Symptoms-? Motor as Well. }
$$

Syringomyelia.

Disseminated sclerosis.

Compression of the cord.

On these lines we are electing to make the sensory symptoms our guide, and therefore it is to the sensory phenomena that we must at once turn our attention. If any are found, we can straightway throw over our first list and concentrate on the second. Per contra, in their absence, the first list is the one to follow up. Let us take each in turn, beginning with the purely motor affections.

* This lymphocytosis is, of course, also associated with the chronic
A. Chronic Spinal Affections with Motor Symptoms Only.

With these the first thing to look for is muscular atrophy; particularly in the little muscles of either hand, or, if not here, about the shoulders or in the legs. Suppose this found, then we are dealing with either progressive muscular atrophy or amyotrophic lateral sclerosis." The distinguishing points between these two can be briefly summarized.

Progressive Muscular Atrophy.-The muscles of the patient's hand and, perhaps, his deltoid and scapular muscles are wasted and show fibrillary contractions. As a result the corresponding reflexes are feeble or lost and the electrical excitability decreased or absent. In a more advanced case the same process may have spread to the patient's legs or, ultimately, to his bulb. Sometimes this sequence is changed, the legs going first, the arms later, but never the bulb (Charcot-Marie-Tooth type). Sometimes, when only the right hand is affected, we have the "occupational" atrophy of file-cutters, gun-barrel engravers and others who, in their employment, make special use of their thenar, hypothenar, and interosseous museles.

Aniyotrophic Lateral Sclerosis.-Here again the patient's hand museles are the first to go, but although the same fibrillary twitching and electrical changes are found his wrist-jerk and triceps-jerk are exaggerated. In addition, his legs gradually becoine rigid and present the features of sclerosis of the lateral columns (spastic gait, exaggerated knee-jerks, ankle clonus, and the Babinski or dorsal plantar reflex). This affection, like the last, sometimes spreads in the late stages up to the bulb.

Suppose, however (the case still being one with only motor symptoms), that no atrophy is to be seen. We have then to decide between primary spastic paraplegia, Friedreich's ataxy, and ataxic paraplegia.

Primary Spastic Paraplegia (or, from the tract affected. in the cord, Primary Lateral Sclerosis).-This is marked off from both the others by the absence of inco-ordination. The patient complains of nothing but his legs, and these show the signs, just mentioned, of lateral sclerosis.

Fricdreich's Ataxy.-The patient, usually a child, and often with close relatives similarly affected, is unable to co-ordinate his legs (and, later, his arms and head) and his knee-jerks are absent. In addition, his speech is peculiarly hesitating. Further, he may have nystagmus.

Ataxic Paraplegia.-Here inco-ordination is present, but neither nystagmus nor any affection of the speech. Moreover the patient's knee-jerks are exaggerated, and he shows both ankle clonus and Babinski's sign.

\section{B. Sensory Symptoms Present, with or without} Motor Symptoms.

We now come to a group with lesions that are rather variable. For this reason, be it noted, the symptoms in each instance may not always conform to one type. Still, allowing for this, we ought to be able to identify every sensory case with one of the following four:

Tabes.-This is too well known to need much by way of description. Any patient without his knee-jerks, but with Argyll Robertson pupils and lightning pains, is beyond question tabetic. In addition to these "pre-ataxic" symptoms, he may show hypotonus of his muscles, permitting extraordinarily free movements of his limbs, very variable sensory changes (including anaesthesia, girdle pains, visceral crises, and optic atrophy), squint, diplopia, and Charcot's joints. Later, when his ataxy has come to the front, his sensory changes may become even more extensive.

Syringomyelia, fully developed, combines the symptoms of (1) progressive muscular atrophy in the arms with (2) spastic paraplegia in the legs, and it might therefore pass as amyotrophic lateral sclerosis, were it not for its remarkable sensory changes. By these the patients are made insensitive to pain and heat and cold. $\uparrow$ Their touch and muscle sense, however, are keen enough. On the other hand, they are subject to trophic changes, particularly glossy skin, cracked nails, or Charcot's joints. .The diagnosis, therefore, turns mainly on the sensory symptoms, and is the easier to make, since these are often the first in the field.

An affection which, in mediaeval times, by enabling people to pick up live coals, and do other things of the sort, was liable to be diasnosed and treated as witcheraft or wizardry. 
In disseminated sclerosis, where the symptoms are particularly variable on account of the sclerosed areas boing so inconstant in their distribution, the patient's legs are weak and spastic with all the tendon-reflexes exaggerated, while his (or her) arms, also weak, may be paraesthetic. More striking than this, however, and more important in the diagnosis, are its three cardinal symptoms, tremor, nystagmus, and staccato speech. By these it may be known, although in the earlier stages, before these three have fully developed, a hysterical counterfeit may lead us astray.

Compression of the cord, the result usually of caries of the spine, an aneurysm or a secondary growth, produces $t$ wofold symptoms - those of spinal root irritation followed by root paralysis, and those of a transverse lesion of the cord itself. Apart from these, however, we may expect a third set due to whatever is causing the compression. If this last group is not to be found, we are then thrown back on the possibility of some primary new growth inside the spinal canal, or a patch of chronically thickened meninges ; in either case, the site of the pressure is identified by the distribution of the symptoms. The distinction between the two, however, may be a matter of difficulty. Perhaps the most suggestive differences are that in chronic meningitis the course is slower and that the pain, even from the beginning, is bilateral and spreads over a wider area. Moreover, the meningitis may improve without surgical treatment.

\section{REMOVAL OF EXTRAMEDULLARY TUMOUR OF CERVICAL CORD : RECOVERY.}

(AREA OF SUPPLY OF EIGHTH CERIICAT AND FIRST DORSAL POSTERIOR ROOTS.)

BY

J. MICHELL CLARKE, M.A., M.D.Cantab., F.R.C.P., JOINT PROFESSOIR OF MEDICINI, DNIVERSITY OF BRISTOL; PHYSICIAN TO THE BRISTOL GENERAL HOSPITAL.

Is connexion with the following case, it is permissible to recall the frequency with which extramedullary tumours of the spinal cord are benign in character, and that this holls good when the patient is in later life. Hence the great importance of diagnosis, for by leading to operation it enables a patient sutfering from one of the most painful and disabling of diseases to be restored to ease and active life. It is as well, also, to bear in mind the great power of recuperation possessed by the spinal cord, so that it can recover its functions even after very severe and longcontinued compression, when the pressure is removed.

L. D., aged 55, married, was sent to the Bristol General Hospital by Dr. Harvey Bird, of Bridgwater, on May 2nd, 1911. Family history good.

The patient had small-pox when a child, and fifteen years ago had exophthalmic goitre for about five years. She theu complete!y recovered, and shows no sign of this disease now, except. perhaps, that her pulse is habitually rather quick84 to 90 per minute. The menopause occurred at 45 years.

\section{History of Symptoms.}

1. Pain.-Two years ago (1909), following influenza, attacks of "cutting;" pain in left shoulder, lasting a few weeks and then "cutting" pain in left shoulder, lasting a few weeks and then disappearing, and worse in damp or cold weather, occurred. Dull aching pains in the left arm followed shortly after those in the shoulder. For a time in 1910 she was much better, though not free from pain at intervals. In November, 1910, she had a fresh attack of " influenza," with severe vomiting and diarrhoea; since then pains in upper part of left chest, left
shoulder, and shooting down left arm to fingers, especially little and ring fingers, have been more or less constant, varvin: in severity, and often preventing sleej. February, 1911, girdle pain around waist.

2. Paraesthesia.-About May, 1910, numbness in left arm from elbow to wrist, around waist, and in her feet and legs. The n'umbness of legs "felt as if it were in patches"; that around the waist disappeared again; in the other parts it remained permanent. Later, numbness and "pins and needles" affected vernar border of left hand and little and ring fingers.

3. Muscular Wusting. - First noticed in left hand and forearm 3. Muscular Wasting. - First noticed in left hand and forearm
after influenza in November, 1910, then improved; but in after influenza in November, 1910, then improved; but in affecting all muscles of hand, and also anterior aspect of forearm. Slight wasting of right hand muscles during last month.

4. Paralysis.-Loss of power in left shoulder, twelve months, probably at first due to pain; she kept her shoulder at rest because movement increased pain. Gradually increasing paralysis of left arm and hand. For two months left hand and forearm almost completely paralysed and useless. Partial paralysis right hand, one month. Gradual loss of power in legs, two months; unable to walk or to stand without support, two weeks; unable to sit up in bed, one week. Muscularcramps in legs not marked.

5. Sphincters.-Difficulty in micturition, four months; worse, three weeks; chief difficulty is in starting micturition. Obstinate and increasing constipation for about same time. General health good; all symptoms have progressed rapidly since shock of husband's sudden death three months ago.

\section{State on Admission.}

Well nourished, rather anaemic, with a distressed expression. She could not walk, nor could she stand without being held up. She could just sit up in bed with support. There was no paralysis of any crania! nerve. The pupils were equal, of paralysis of any cranial nerve. The pupils were equal, of painful stimulation of skin of neck. The neck muscles were painful stimu

Upper Extremities.-Right: Grip of hand very weak, moderate legree of wasting of small muscles of hand; arm and forearm unaffected. The hand muscles reacted to faradism, but required a strong current. No affection of sensation. Left: Small muscles of hand uniformly and extremely wasted, including muscles of thenar and hypothenar eminences, dorsal interossei and lumbricales. The muscles did not react to a strong faradic current. The little and ring fingers were The little and ring at proximal and flexed at distal joints. completely completely lisalysed and useless. The long flexors of forearm were paralysed and did not react to faradism; the extensors were not paralysed and reacted. The elbow could not be fully extended. She could not raise her arm above the shoulder; movements of the shoulder were generally limited, and the shoulder muscles contracted, especially the pectorals, but not much wasted, from disuse owing to pain, and there were adhesions about the shoulder joint. Partial loss of sensation over inner (post-axial) side of limb. (See Fig. 1.) The elbow
and wrist jerks were present on both sides. No loss of sense of and wrist jerks were present on both sides. No loss of sense of
position of either hand or arm, and objects placed in hands recognized at once.

Spinc.-Some kyphosis in dorsi-lumbar region and slight lateral curvature.

Abdomen.-Apparently defective sensation to pin-prick over abdomen. Abdominal reflexes absent.

Lower Extremities.-The legs could be drawn up but not lifted off the bed. There was marked spasticity, the muscles being in a state of active spasm, but not wast 3 . Knee-jerks much exaggerated; slight ankle clonus on right side, not on left. Plantar reflex, extensor and Oppenheim's and paradoxical reflexes present on both sides.

Sphincters.-Complete retention of urine, which was acid, and contained no albumen or sugar. Obstinate constipation. Thoracic and abdominal organs normal.

Wassermann's reaction negative. Tuberculin test negative. Cercbro-spinal fluid appeared under slightly diminished pres-
sure and contained a considerably increased number of sure and contained a considerably
cytes, but was otherwise normal.

cytes, but was otherwise normal.
It was considered advisable to give her a course of potassium iodide with liq. hydrarg. perchlor. This she had.

\section{Condition on Jume 14th (Five Weeks after Admission).}

She had a great deal of pain during this time in the left shoulder, passing down into the arm, and painful spasmodic contractions of left shoulder miuscles and those of lower part of neck. Pain was increased both by passive and active movements. Pains had also begun to affect the right shoulder and arm. They were never so intense as to cause her to cry out, but arm. They were neve

Wasting of small muscles of the hand was complete on left side and had rapidly advanced on right. There was also wasting of muscles on anterior surface of left forearm, and to a less degree of the right. The left upper extremity was completely paralysed. She could not hold a pen or spoon in the right hand, fore useless, and she had to be fed. Except that she could draw up the legs a few inches in bed, they were also paralysed. She could not sit up or turn in bed. Except for movements of the head and neck, of the right shoulder and arm, and, to a less degree, of the right forearm and of those muscles supplied by the cranial nerves, she was therefore completely paralysed. The pupils were unaffecterl. There was complete retention of urine and incontinence of faeces. Some defect of sensation to pain and temperature had now appeared over trunk and legs, and there was some loss of sense of position of the left arm and of the legs. The condition of the reflexes was the same as above stated.

After this period of observation the diagnosis was arrived at of tumour of the cord, external to it, on the left side, and at the level of, and compressing, the eighth cervical and first dorsal roots. I therefore asked my colleague, Mr. Hey Groves, to see the case, and he operated on June 2lst.

Solution of adrenalin, 1 in 50,000 .

She skin and muscles. The laminae of the sixth and seventh cervical and first dorsal. The laminae were removed on the left side 